\title{
Nonlinear Dynamics of Vertical-Cavity Surface-Emitting Lasers
}

\author{
S. F. Yu
}

\begin{abstract}
The dependence of the transient response of a vertical-cavity surface-emitting laser (VCSEL) on its aperture size is investigated subject to direct current modulation and external optical feedback. It is shown that lasers with small aperture size suppress higher order bifurcations and chaos even under large-signal modulation and external optical feedback. Furthermore, the noise characteristics of VCSEL's under the influence of external optical feedback are studied via the calculation of relative intensity noise. It is found that the level of external optical feedback for the onset of coherence collapse is high for devices with small aperture size. On the other hand, the smallsignal response of lasers is also analyzed through the calculation of third-order harmonic distortion. It is shown that harmonic distortion is minimized in small devices. Therefore, VCSEL's with small aperture size have better immunity to irregular response under direct current modulation and external optical feedback.
\end{abstract}

Index Terms - Bifurcation diagrams, chaos, direct modulation, external optical feedback, semiconductor device modeling, surface-emitting devices.

\section{INTRODUCTION}

V ERTICAL-CAVITY surface-emitting lasers (VCSEL's) are promising for applications in optical communication and interconnects because of their useful characteristics such as low threshold current, single-mode operation, circular output beam, and wafer-scale integration. Recently, studies have concentrated on the steady state and small-signal response of VCSEL's with different aperture sizes [1]-[3]. It is found that the threshold current and light-current curves of a VCSEL are determined by its cavity loss and thermal resistance, which are size-dependent [1], [2]. Furthermore, small-signal analysis shows that a VCSEL with a small aperture size exhibits wider modulation bandwidth and less second harmonic distortion due to its high relaxation oscillation frequencies [2], [3]. It is believed that higher order harmonic distortion can be minimized in VCSEL's with small aperture size.

Facet emitted lasers under large signal modulation may exhibit various kinds of irregular response such as period doubling, period quadrupling, and chaos [4]-[9]. However, these nonlinear characteristics are suppressed in devices with large spontaneous emission factor $\left(>10^{-5}\right)[6]$, [9]. Hence, a VCSEL with a small aperture size can have strong immunity to higher order bifurcations and chaos even under large-signal modulation due to its inherent large value of spontaneous emission factor. This is because the coupling efficiency be-

Manuscript received March 25, 1998; revised November 24, 1998. The author is with East Harbour Telecommunication, Ltd., Hong Kong. Publisher Item Identifier S 0018-9197(99)01732-7. tween the spontaneous emission and cavity mode is enhanced by the quantum electrodynamic effects inside the microcavity, especially for devices with small aperture size [10]-[12]. To the best of our knowledge, no evidence on the chaotic behavior of VCSEL's has been reported. Therefore, it is expected that the large-signal response of VCSEL's also depends on its aperture size.

The noise characteristics of lasers subject to external optical feedback are dependent on the energy stored inside the laser cavity and the coupling of the laser mode to the external field. It is believed that the transient to coherence collapse can be suppressed in VCSEL's with small aperture size due to the high storage of photon density inside the laser cavity [13]-[15]. Therefore, it is interesting to study the noise characteristics of VCSEL's with different aperture sizes subject to external optical feedback.

A number of theoretical models have been developed to analyze the transient response of VCSEL's under the influence of external optical feedback [16]-[18]. However, neither the relative importance of size-dependent parameters nor the influence of direct current modulation has been taken into account in these calculations. Therefore, the aim of this paper is to investigate the dependence of the transient response of a VCSEL on its aperture size subject to direct current modulation and external optical feedback. In Section II, a rateequation model is developed with size-dependent parameters such as thermal resistance, cavity, and spontaneous emission factor taken into the calculation. In Sections III and IV, the modulation response of VCSEL's subject to large-signal modulation and external optical feedback is analyzed. In Section V, the correlation between aperture size and the onset of coherence collapse under the influence of external optical feedback is studied. In Section VI, the dependence of thirdorder harmonic distortion on the aperture size of VCSEL's is also evaluated. The results are briefly discussed and the conclusions are given in Section VII.

\section{LASER PARAMETERS AND MODEL}

\section{A. Laser Structure and Model}

The schematic of an index guiding VCSEL used in the analysis is shown in Fig. 1. The device structure is similar to that given in [2] except for the p-type distributed Bragg reflector, which is surrounded by a cladding layer. The reflectivities of $\mathrm{n}$ and p-type Bragg reflectors are assumed to be equal to $R_{0}$ and $R_{1}$, respectively. The corresponding single-mode rate-equation 


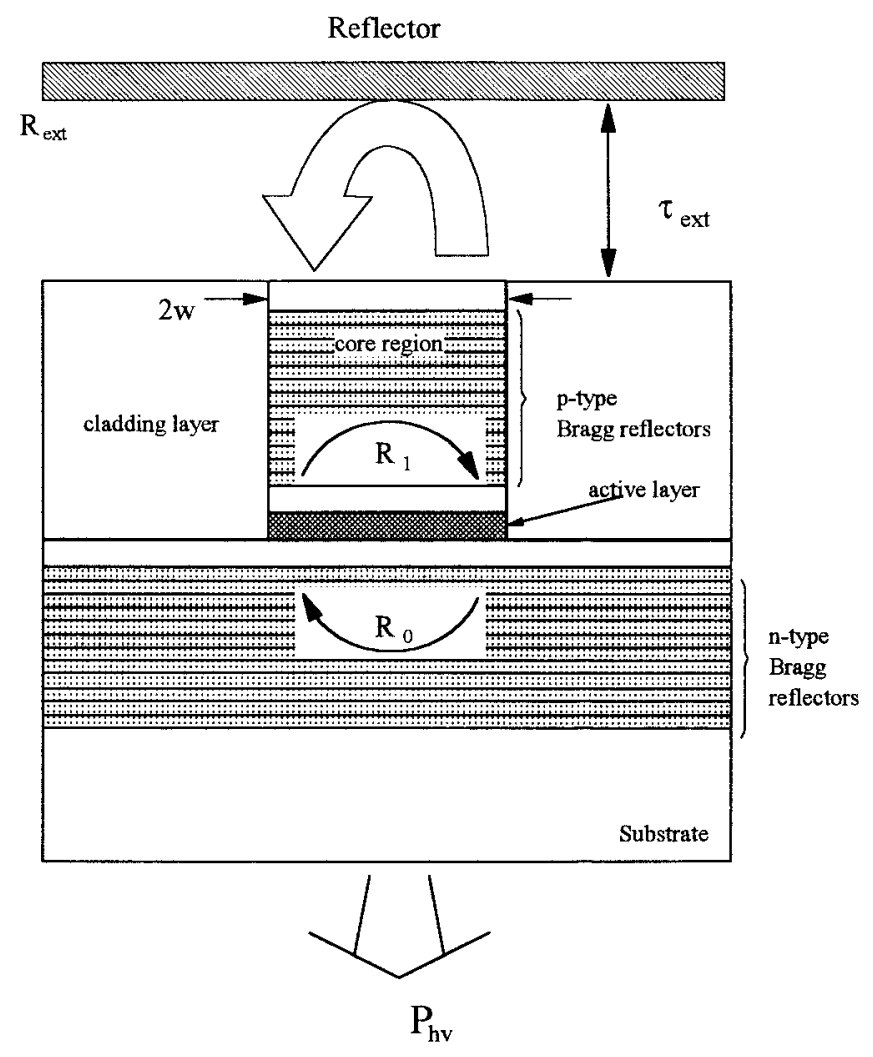

Fig. 1. Schematic of an index-guided VCSEL used in the calculation.

model of VCSEL's is given by [3]

$$
\begin{aligned}
\frac{\partial P(t)}{\partial t}= & \nu_{g}(\Gamma G(t)-\alpha) P(t)+\beta B_{\mathrm{sp}} N^{2}(t)+F_{p}(t) \\
& +\operatorname{Re} f_{p}(t) \\
\frac{\partial N(t)}{\partial t}= & \frac{I_{s}(t)}{q V}-\nu_{g} \Gamma G(t) P(t)-\frac{N(t)}{\tau_{s}}+F_{N}(t) \\
\frac{\partial T(t)}{\partial t}= & \frac{1}{C_{\mathrm{th}}}\left(P_{I V}(t)-P_{h v}(t)-\kappa \frac{T(t)-T_{0}}{R_{\mathrm{th}}}\right)+F_{T}(t) \\
\frac{\partial \phi(t)}{\partial t}= & \frac{1}{2} \alpha_{H} \nu_{g} \Gamma \frac{\partial G}{\partial N}\left(N(t)-N_{t h}\right)+F_{\phi}(t)+\operatorname{Re} f_{\phi}(t) \\
I_{s}= & I_{\mathrm{dc}}+I_{r f} \sin \left(2 \pi f_{m} t\right)
\end{aligned}
$$

where $P$ is the photon density, $N$ is the carrier concentration, $T$ is the effective temperature, and $\phi$ is the phase. $N_{\text {th }}$ is the threshold carrier concentration, $\alpha_{H}$ is the linewidth enhancement factor, and $\partial G / \partial N$ is the differential gain. The injection current $I_{s}$ is the sum of the dc bias current $I_{\mathrm{dc}}$ and sinusoidal modulation current, $I_{r f} \sin \left(2 \pi f_{m} t\right) . I_{r f}$ and $f_{m}$ are the amplitude and modulation frequencies, respectively, of the sinusoidal modulation current.

In the photon rate equation, $\Gamma$ is the lateral confinement factor, $\alpha$ is the equivalent cavity loss, $\nu_{g}$ is the group velocity, and $B_{\mathrm{sp}}$ is the bimolecular carrier recombination factor. The dependence of spontaneous emission factor $\beta$ on the cavity size can be evaluated by [12]

$$
\beta=\frac{3}{2} \frac{\Gamma_{z} K \lambda_{0}^{4}}{4 \pi^{2} n_{\mathrm{eff}}^{3} V \Delta \lambda}
$$

TABLE I

Temperature-IndePendent PaRAmeters of Optical Gain

\begin{tabular}{c|c}
\hline Symbol & Value \\
\hline \hline $\mathrm{a}_{0}$ & $-46963.3 \mathrm{~cm}^{-1}$ \\
\hline $\mathrm{a}_{1}$ & $371.56 \mathrm{~cm}^{-1} \mathrm{~K}^{-1}$ \\
\hline $\mathrm{a}_{2}$ & $-0.941029 \mathrm{~cm}^{-1} \mathrm{~K}^{-2}$ \\
\hline $\mathrm{a}_{3}$ & $7.99274 \times 10^{-4} \mathrm{~cm}^{-1} \mathrm{~K}^{-2}$ \\
\hline $\mathrm{b}_{0}$ & $2.723 \times 10^{18} \mathrm{~cm}^{-3}$ \\
\hline $\mathrm{b}_{1}$ & $-2.417 \times 10^{16} \mathrm{~cm}^{-3} \mathrm{~K}^{-1}$ \\
\hline $\mathrm{b}_{2}$ & $6.4786 \times 10^{13} \mathrm{~cm}^{-3} \mathrm{~K}^{-2}$ \\
\hline
\end{tabular}

where $\lambda_{0}$ is the lasing wavelength, $\Delta \lambda$ is the full-width at half-maximum (FWHM) of the emission spectrum, $K$ is Petermann's astigmatism factor, $\Gamma_{z}$ is the longitudinal confinement factor, $n_{\text {eff }}$ is the effective refractive index of the cavity, $V\left(=\pi W^{2} d\right)$ is the volume of the active layer, $d$ is the active region thickness, and $W$ is the core radius. The equivalent optical gain $G$ can be expressed as

$$
G(t)=\Gamma_{z} a_{N} \frac{\log \left[(N(t)-0.41) / N_{0}\right]}{1+\varepsilon P(t)}
$$

where $\varepsilon$ is the gain compression factor. The parameters $a_{N}$ and $N_{0}$ are assumed to be temperature-dependent and can be approximated by $a_{N}=a_{0}+a_{1} T+a_{2} T^{2}+a_{3} T^{3}$ and $N_{0}=b_{0}+b_{1} T+b_{2} T^{2}$ (see also Table I).

In the carrier rate equation, the carrier lifetime $\tau_{s}$ can be expressed as

$$
\tau_{s}=\frac{\tau_{s 0}}{\exp \left(\left(T(t)-T_{0}\right) / T_{0 s s}\right)}
$$

where $\tau_{s 0}$ is the carrier lifetime at $300 \mathrm{~K}, T_{0}$ is the background temperature, and $T_{0 s s}$ is the characteristic temperature.

In the thermal rate equation, $\kappa$ is the thermal conductivity and $R_{\mathrm{th}}$ is the thermal resistance. The thermal capacitance $C_{\mathrm{th}}$ of the device can be expressed as

$$
C_{\mathrm{th}}=\rho_{m} C_{p} \pi W^{2} d
$$

where $\rho_{m}$ is the mass density and $C_{p}$ is the thermal conductivity. The output power $P_{h v}$ of the device is given by

$$
P_{h v}=\frac{1}{2} \nu_{g} h v\left(1-\left|R_{0}\right|^{2}\right) \pi W^{2} \Gamma_{z} P(t)
$$

where $h$ is the Planck constant and $\nu$ is the frequency of the lasing mode. The total input electrical power $P_{T V}$ is defined as

$$
P_{T V}=\frac{1}{2} V_{F}(N) I_{s}
$$

where $V_{F}$ is the voltage across the active layer and is approximated by

$$
\begin{aligned}
V_{F}= & {\left[E_{g}+k_{B} T \cdot \log \left\{\left(\exp \left(N / N_{c}\right)-1\right)\right.\right.} \\
& \left.\left.\cdot \exp \left(\left(N / N_{v}\right)-1\right)\right\}\right]
\end{aligned}
$$

where $k_{B}$ is the Boltzmann constant and $E_{g}$ is the energy gap between the first quantized energy level of conduction and the 
valence bands of the quantum well's active layer. $N_{c}$ and $N_{v}$ are the effective conduction and valence edge density of states, respectively, and can be expressed as

$$
N_{c / v}=m_{e / h}^{*} k_{B} T / \pi \hbar^{2} L_{z}
$$

where $L_{z}(=80 \AA)$ is the thickness of the quantum wells, $\hbar=h / 2 \pi$, and $m_{e / h}^{*}(=0.0665 / 0.33196)$ is the effective mass of electrons/holes.

The parameters $F_{P}, F_{N}, F_{T}$, and $F_{\phi}$ are the Langevin noise sources of photon, carrier, temperature, and phase, respectively. The corresponding Langevin noise sources are given by

$$
\begin{aligned}
F_{p}(t) & =\sqrt{\frac{2 P\left(t_{i}\right) \beta N(t) \Gamma_{z}}{\tau_{s} \Delta t}} x_{p} \\
F_{N}(t) & =-\sqrt{\frac{2 P\left(t_{i}\right) \beta N(t) \Gamma_{z}}{\tau_{s} \Delta t}} x_{p}+\sqrt{\frac{2 N\left(t_{i}\right)}{\tau_{s} \Delta t V}} x_{N} \\
F_{T}(t) & =\sqrt{\frac{T\left(t_{i}\right)\left(P_{I V}(t)-P_{h v}(t)\right)}{\kappa C_{t h} \Delta t}} x_{T} \\
F_{\phi}(t) & =\frac{1}{P(t)} \sqrt{\frac{P\left(t_{i}\right) \beta N(t) \Gamma_{z}}{2 \tau_{s} \Delta t}} x_{\phi},
\end{aligned}
$$

where $P\left(t_{i}\right)$ and $N\left(t_{i}\right)$ are the photon density and carrier concentration, respectively, at the start of the time interval $\Delta t$, and $x_{N}, x_{P}, x_{T}$, and $x_{\phi}$ are Gaussian distributed random variables with zero mean and unity variance.

The optical feedback in the rate equations of photon density and phase, $\operatorname{Re} f_{p}$ and $\operatorname{Re} f_{\phi}$, are given by

$$
\begin{aligned}
& \operatorname{Re} f_{p}(t)=\frac{k_{\text {ext }}}{\tau_{L}} \sqrt{P(t)} \sqrt{P\left(t-\tau_{\text {ext }}\right)} \cos (\theta(t)) \\
& \operatorname{Re} f_{\phi}(t)=-\frac{k_{\text {ext }}}{\tau_{L}} \frac{\sqrt{P\left(t-\tau_{\text {ext }}\right)}}{\sqrt{P(t)}} \sin (\theta(t))
\end{aligned}
$$

where $\theta(t)=\phi(t)-\phi\left(t-\tau_{\text {ext }}\right)+\omega_{\text {th }} \tau_{\text {ext }}, \omega_{\text {th }}$ is the oscillation frequency at threshold, $\tau_{\text {ext }}$ is the external round-trip delay time, and $\tau_{L}$ is the round-trip time inside the laser cavity. The feedback parameter $k_{\text {ext }}$ is defined as

$$
k_{\text {ext }}=\eta\left(1-R_{1}\right) \sqrt{R_{\text {ext }}} / \sqrt{R_{1}}
$$

where $R_{\text {ext }}$ is the external reflectivity and $\eta$ is the coupling between the external mirror and the laser. The relaxation oscillation frequency of VCSEL's $f_{r}$ can be written as

$$
f_{r}^{2}=\frac{1}{4 \pi^{2}} \frac{\nu_{g} P_{s}}{\tau_{\mathbf{p h}}}\left(\frac{1}{1+\varepsilon P_{s}} \frac{\Gamma_{z} a_{N}}{N_{s}}\right)
$$

where $\tau_{\mathrm{ph}}=1 / \nu_{g} \alpha$ and $N_{s}$ and $P_{s}$ are the steady-state carrier concentration and photon density, respectively.

\section{B. Steady-State Characteristics of VCSEL's}

Fig. 2 shows the measured light-current curves and relaxation oscillation frequencies $f_{r}$ of VCSEL's with different aperture size $W$. The corresponding calculated results are also plotted on the diagrams. The experimental results match with

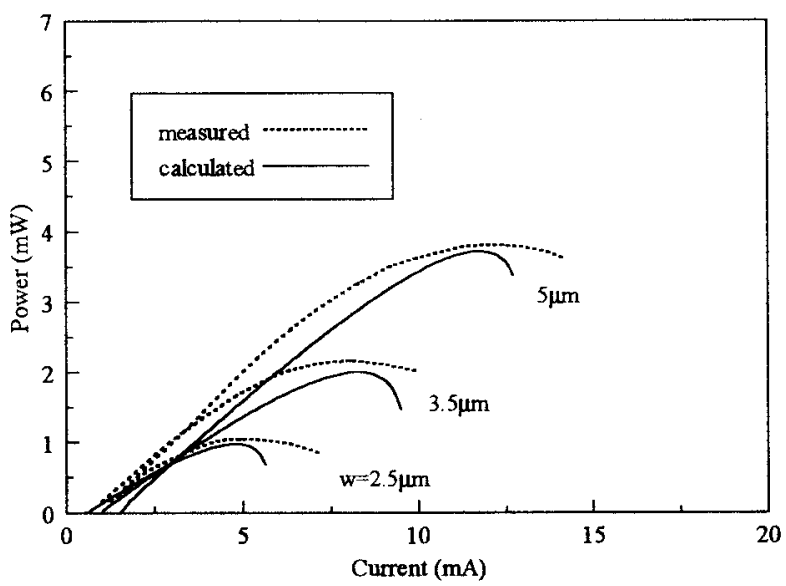

(a)

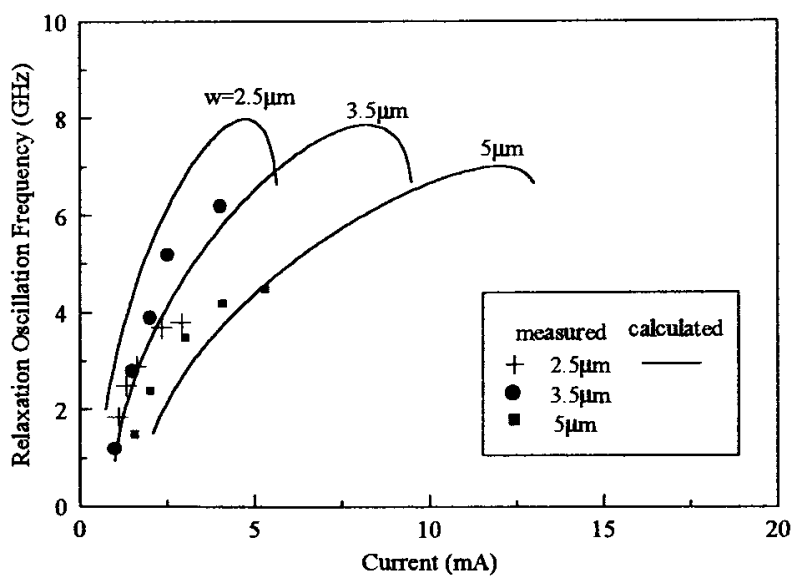

(b)

Fig. 2. Comparison of measured and calculated (a) light-current characteristics and (b) relaxation oscillation frequency of VCSEL's with core radii equal to $2.5,3.5$, and $5 \mu \mathrm{m}$.

the theoretical model using (1)-(3) with $R_{\mathrm{th}}$ and $\alpha$ as the variable parameters. The values of $R_{\mathrm{th}}$ and $\alpha$ as well as other laser parameters used in the calculation can be found in Tables II and III. Fig. 2(a) compares the light-current curves obtained from the theoretical model and the experimental results [2]. It is noted that the model using (1)-(3) does not fit well when compared with the model given in [3]. This indicates that carrier transport and spatial hole burning have a significant influence on the steady-state characteristics of VCSEL's. Nevertheless, the threshold current, the slope of the curves, and the peak power calculated from the model match with the measured data. Fig. 2(b) compares $f_{r}$ for VCSEL's calculated from the model with that obtained from the experimental results. As we can see, $f_{r}$ calculated from the model is close to the experimental results except for the device with $W=2.5 \mu \mathrm{m}$. This may be attributed to the high photon density that drives the optical gain toward the saturation level of the quantum wells which reduces the differential gain and $f_{r}$.

\section{Simulation Results-Modulation RESPONSE}

In this section, the influence of $W$ on the modulation response of VCSEL's is analyzed by varying the modula- 
TABLE II

Size-DEPENDENT PARAMETERS

\begin{tabular}{c|c|c|c}
\hline $\mathrm{W}(\mu \mathrm{m})$ & $\alpha\left(\mathrm{cm}^{-1}\right)$ & $\mathrm{R}_{\mathrm{th}}\left(\mathrm{cm}^{-1}\right)$ & $\beta$ \\
\hline \hline 2.5 & 73 & 7100 & $10^{-3}$ \\
\hline 3.5 & 64 & 4200 & $5 \times 10^{-4}$ \\
\hline 5 & 50.3 & 2900 & $10^{-4}$ \\
\hline
\end{tabular}

TABLE III

PARAMETERS OF DEVICES

\begin{tabular}{l|l|l}
\hline Parameter & Symbol & Value \\
\hline \hline lasing wavelength & $\lambda_{0}$ & $980 \mathrm{~nm}$ \\
\hline FWHM of emission spectrum & $\Delta \lambda$ & $37 \mathrm{~nm}$ \\
\hline effective refractive index of cavity & $\mathrm{n}_{\mathrm{eff}}$ & 3.3 \\
\hline Petermann's astigmatism factor & $\mathrm{K}$ & 1 \\
\hline thickness of active region & $\mathrm{d}$ & $0.1 \times 10^{-4} \mathrm{~cm}$ \\
\hline group velocity & $\nu_{\mathrm{B}}$ & $0.81 \times 10^{10} \mathrm{~cm} / \mathrm{s}$ \\
\hline lateral confinement factor & $\Gamma$ & 1.0 \\
\hline longitudinal optical confinement factor & $\Gamma_{\mathrm{z}}$ & 0.07 \\
\hline gain suppression factor & $\varepsilon$ & $1 \times 10^{-17} \mathrm{~cm}^{3}$ \\
\hline carrier lifetime in active layer & $\tau_{\mathrm{s} 0}$ & $2.7 \mathrm{~ns}$ \\
\hline background temperature & $\mathrm{T}_{0}$ & $300^{\circ} \mathrm{K}$ \\
\hline characteristic temperature & $\mathrm{T}_{0 \mathrm{ss}}$ & $30^{\circ} \mathrm{K}$ \\
\hline bimolecular recombination coefficient & $\mathrm{B}_{\mathrm{sp}}$ & $1 \times 10^{-10} \mathrm{~cm}^{6} \mathrm{~s}^{-1}$ \\
\hline mass density & $\mathrm{P}_{\mathrm{m}}$ & $5.36 \mathrm{~g} / \mathrm{cm}^{3}$ \\
\hline specific heat & $\mathrm{C}_{\mathrm{p}}$ & $0.35 \mathrm{Jg} \mathrm{g}^{-1} \mathrm{C}^{-1}$ \\
\hline thermal conductivity & $\kappa$ & $0.45 \mathrm{Wcm} \mathrm{c}^{-1} \mathrm{C}^{-1}$ \\
\hline linewidth broadening factor & $\alpha_{\mathrm{H}}$ & 4.8 \\
\hline laser to fiber coupling efficiency & $\eta$ & 0.4 \\
\hline reflectivity of $\mathrm{n}$-type Bragg reflector & $\mathrm{R}_{0}$ & 0.997 \\
\hline reflectivity of p-type Bragg reflector & $\mathrm{R}_{\mathrm{l}}$ & 0.99 \\
\hline laser cavity round trip delay & $\tau_{\mathrm{L}}$ & $0.15 \mathrm{ps}$ \\
\hline external round trip delay time & $\tau_{\mathrm{ext}}$ & $2 \mathrm{~ns}$ \\
\hline relative phase of reflected field & $\omega_{\mathrm{th}} \cdot \tau_{\mathrm{ext}}$ & $2 \pi \times$ integer \\
\hline & &
\end{tabular}

tion index $m\left(=I_{r f} / I_{\mathrm{dc}}\right)$. Furthermore, the influence of sizedependent parameters such as $P_{s}, f_{r}, \beta$, and the self-heating effect on the bifurcation diagrams is investigated. In the following calculation, it is assumed that the steady-state output power of the laser is maintained at $0.32 \mathrm{~mW}$. The modulation frequency $f_{m}$ is set to $4 \mathrm{GHz}$ which is comparable to $f_{r}$ for these devices. The modulation responses of lasers are recorded after the modulation current was applied for $200 \mathrm{~ns}$ in order to eliminate the influence of initial transients. Furthermore, the Langevin noise sources are set to zero in order to observe the fine structure of the bifurcation diagrams. In fact, the bifurcation diagrams are only slightly thickened by the noise sources and the entire profile of the bifurcation diagrams has less deviation from the quiet solutions. It can be shown that devices with large $W$ exhibit period doubling as well as period quadrupling due to the size-dependent parameters of VCSEL's.

\section{A. Influence of Aperture Size on the Modulation Response of VCSEL's}

The modulation response of VCSEL's with different aperture sizes $W$ may demonstrate dissimilar characteristics even with the same output power and modulation conditions. Fig. 3(a) shows the variation of normalized peak photon density $P_{\text {npeak }}\left(P_{\text {npeak }}=P_{\text {peak }} / P_{n}\right.$ where $P_{\text {peak }}$ is the peak photon density and $P_{n}=10^{18} \mathrm{~cm}^{-3}$ is a normalizing parameter) versus $m$ for a device with $W=3.5 \mu \mathrm{m}$. It is

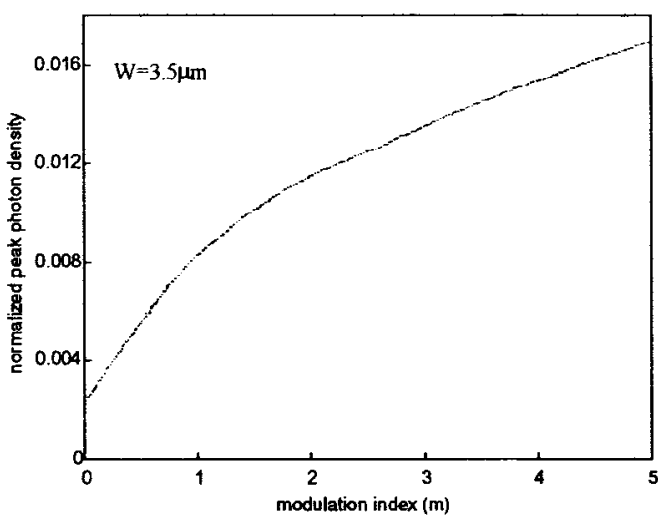

(a)

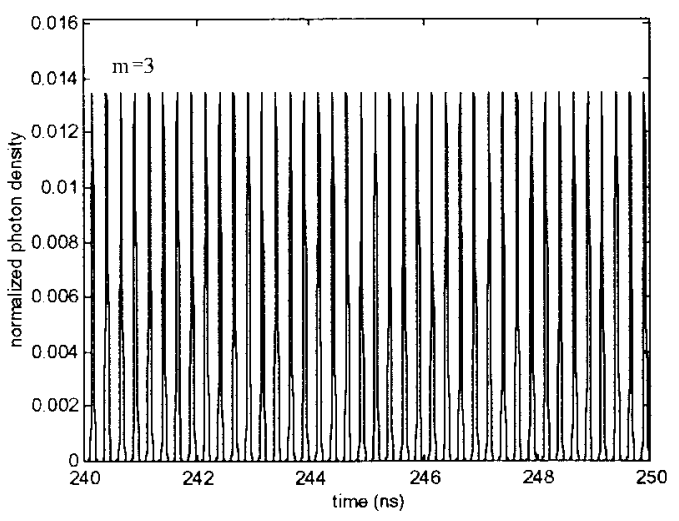

(b)

Fig. 3. Modulation response of VCSEL with $W=3.5 \mu \mathrm{m}$ and $f_{m}=4$ GHz. (a) Bifurcation diagram of normalized peak photon density versus modulation index. (b) Transient response of normalized photon density at $m=3$.

observed that the device exhibits single-period oscillation for the whole range of $m$ (i.e., $0<m \leq 5$ ). The corresponding transient behavior of normalized photon density $P_{\text {norm }}$ $\left(P_{\text {norm }}=P / P_{n}\right)$ at $m=3$ is shown in Fig. 3(b). Furthermore, the bifurcation diagram of $P_{\text {npeak }}$ versus $m$ for a device with $W=5 \mu \mathrm{m}$ is shown in Fig. 4(a). It is observed that, for the range of $m$ between 0.5 and 1.6, period doubling is excited and the corresponding transient behavior of $P_{\text {norm }}$ is shown in Fig. 4(b). Two sets of pulse trains, one with a relatively low and the other with relatively high peak photon density, are observed. For $1.6<m<2.2$, period quadrupling is excited and the corresponding transient behavior of $P_{\text {norm }}$ is shown in Fig. 4(c). However, further increases in $m$ will bring back period doubling and then single-period oscillation.

From the above, it is observed that the bifurcation behavior of VCSEL's is dependent on $W$. In fact, the modulation response of VCSEL's also depends on the size-dependent parameters such as $P_{s}, f_{r}, \beta$, and the self-heating effect.

\section{B. Influence of Photon Density on the Modulation Response of VCSEL's}

It must be noted that the values of $P_{S}$ are equal to $2.4 \times 10^{15}$ and $1.2 \times 10^{15} \mathrm{~cm}^{-3}$ for devices with $W=3.5$ and $5 \mu \mathrm{m}$, respectively. Therefore, it is expected that the bifurcation behavior of VCSEL's is affected by $P_{s}$. Fig. 5 plots the 


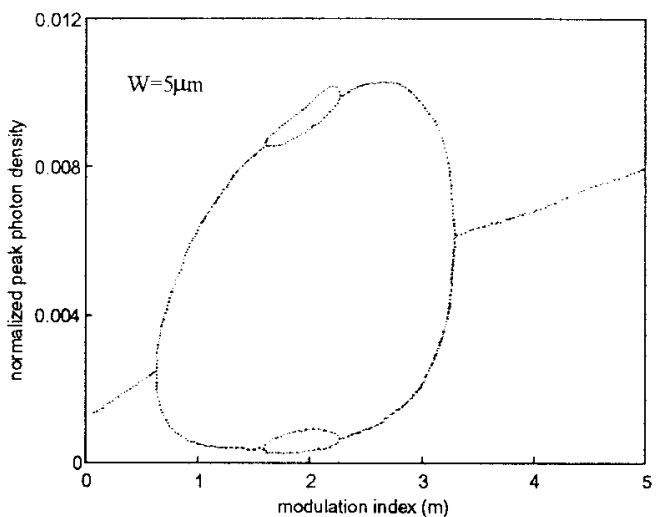

(a)

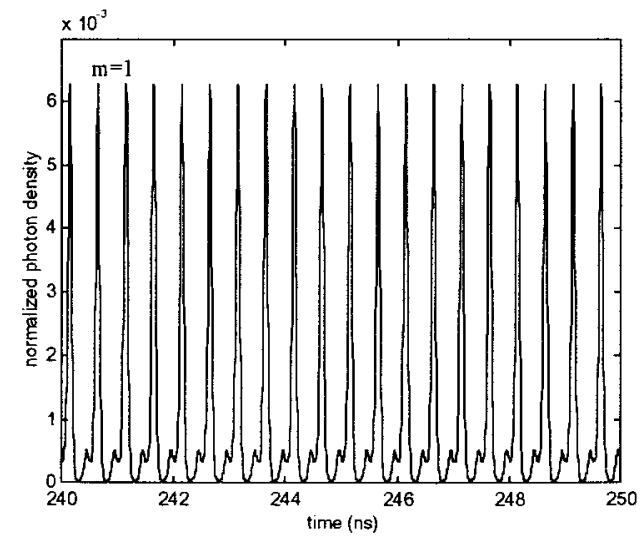

(b)

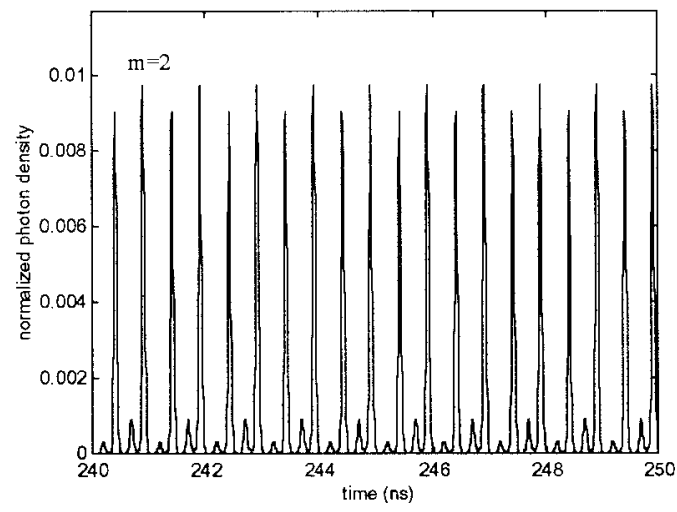

(c)

Fig. 4. Modulation response of VCSEL with $W=5 \mu \mathrm{m}$ and $f_{m}=4 \mathrm{GHz}$. (a) Bifurcation diagram of normalized peak photon density versus modulation index. (b), (c) Transient responses of normalized photon density at $m=1$ and 2 , respectively.

bifurcation diagrams of $P_{\text {peak }}$ versus $m$ for a device with $W=3.5 \mu \mathrm{m}$ and the value of $P_{s}$ varies from $1.2 \times 10^{15}$ to $2.4 \times 10^{15} \mathrm{~cm}^{-3}$. It is observed that period doubling is excited due to the reduction of $P_{s}$. The results given in Fig. 5 are consistent with our previous analysis because a large device (i.e., $5 \mu \mathrm{m}$ ) with low $P_{s}$ enhances higher order bifurcations.

On the other hand, it is noted from (21) that $P_{s}$ is directly proportional to $f_{r}$ such that the bifurcation behavior of the devices is also dependent on $f_{r}$. If $f_{m}$ is the same for VCSEL's with large and small aperture sizes, double or higher order

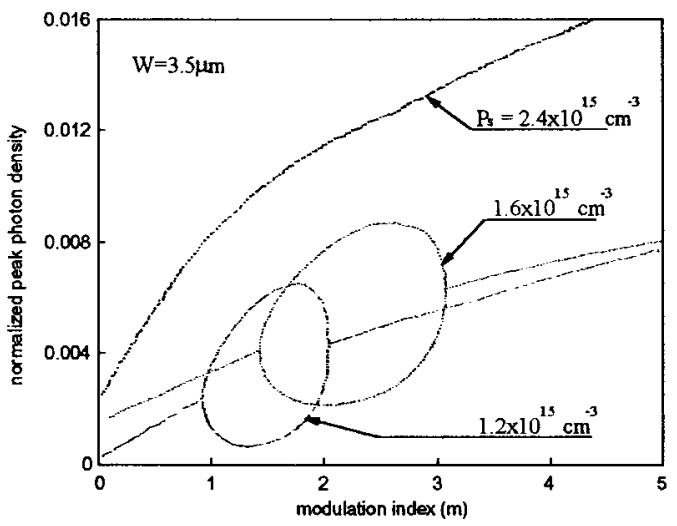

Fig. 5. Influence of photon density $P_{s}$ on the bifurcation diagram of a VCSEL with $W=3.5 \mu \mathrm{m}$ and $f_{m}=4 \mathrm{GHz} . P_{s}$ is varied between $1.2 \times 10^{15}$ and $2.4 \times 10^{15} \mathrm{~cm}^{-3}$.

bifurcations are more likely to be excited in large devices due to small $f_{r}$. However, small devices can maintain a stable single oscillation period even under large signal modulation due to its high $f_{r}$.

\section{Influence of Modulation Frequency on the Modulation Response of VCSEL's}

It must be noted that the bifurcation and chaotic behavior of facet-emitting lasers is enhanced for $f_{m}>f_{r}$ but suppressed for $f_{m}<f_{r}$ [5]. Therefore, it is interesting to know whether VCSEL's also exhibit similar characteristics to that for facetemitting devices.

Fig. 6 repeats the calculation in Fig. 4 but with $f_{m}$ varied between $0.65 f_{r}$ and $1.63 f_{r}$. It is observed that a single-period oscillation is maintained for $f_{m}$ less than $0.7 f_{r}$ but period doubling as well as period quadrupling are excited for $f_{m}$ greater than $f_{r}$. It is noted that period doubling starts at $m=0.49$ for $f_{m}=1.02 f_{r}$ and shifts to $m=1.55$ for $f_{m}=1.63 f_{r}$. Hence, the modulation response of VCSEL's with a large value of $W$ is similar to that of facet-emitting lasers [5]. However, for devices with small $W$ (i.e., $3.5 \mu \mathrm{m}$ ) and the same output power, $f_{m}$ has a negligible influence on the bifurcation behavior of lasers and only a singleperiod oscillation is observed. This is not consistent with the above observation and implies that the bifurcation behavior of VCSEL's may also depend on other size-dependent parameters such as $\beta$.

\section{Influence of Spontaneous Emission Factor on the Modulation Response of VCSEL's}

It is noted that the value of $\beta$ is dependent on the value of $W$ for a VCSEL. Therefore, it is interesting to know whether the dependence of $\beta$ on $W$ has any influence on the modulation response of VCSEL's. Fig. 7 shows the bifurcation diagram of $P_{\text {npeak }}$ versus $m$ for the device with $W=3.5 \mu \mathrm{m}$, and the value of $\beta$ is changed deliberately from $5 \times 10^{-4}$ to $10^{-8}$. It is observed that period doubling is excited for $\beta$ less than $5 \times 10^{-4}$. Further reduction of $\beta$ increases the range of $m$ for period doubling. Fig. 7(b) shows the bifurcation diagram of $P_{\text {npeak }}$ versus $m$ for the device for which $\beta$ equals $10^{-8}$. 


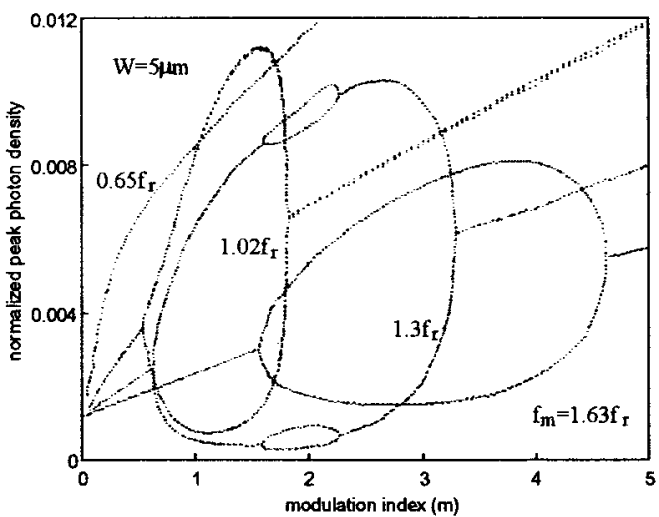

Fig. 6. Influence of modulation frequency $f_{m}$ on the bifurcation diagram of VCSEL's with $W=5 \mu \mathrm{m}$. $f_{m}$ is varied between $0.65 f_{r}$ to $1.63 f_{r}$.

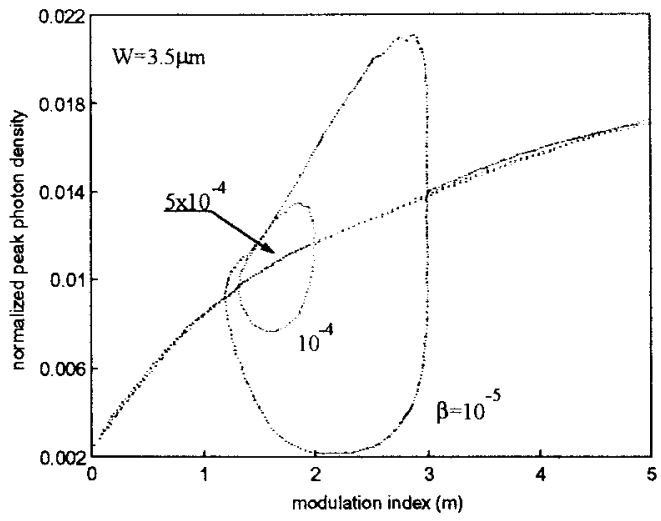

(a)

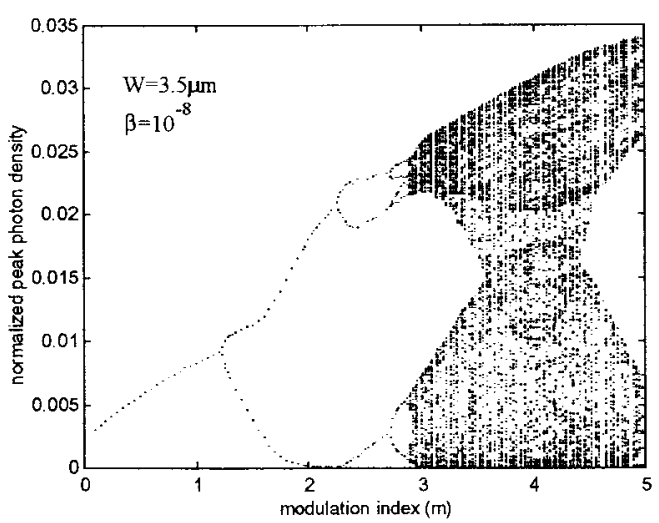

(b)

Fig. 7. Influence of the spontaneous emission factor $\beta$ on the bifurcation diagram of a VCSEL with $W=3.5 \mu \mathrm{m}$ and $f_{m}=4 \mathrm{GHz}$. (a) $\beta$ varies from $10^{-4}$ to $10^{-5}$. (b) $\beta$ is set to $10^{-8}$.

It is observed that single period oscillation, higher order bifurcations, and chaos are excited with an increase of $m$. Hence, it is shown that the bifurcations and chaotic behavior of VCSEL's are also dependent on the value of $\beta$. In addition, the chaotic behavior of VCSEL's under large-signal modulation can be suppressed by using a large value of $\beta$ (i.e., $\geq 5 \times 10^{-4}$ ).

\section{E. Influence of Self-Heating Temperature on the Modulation Response of VCSEL's}

It is expected that the self-heating effect may have an influence on the modulation response of VCSEL's because

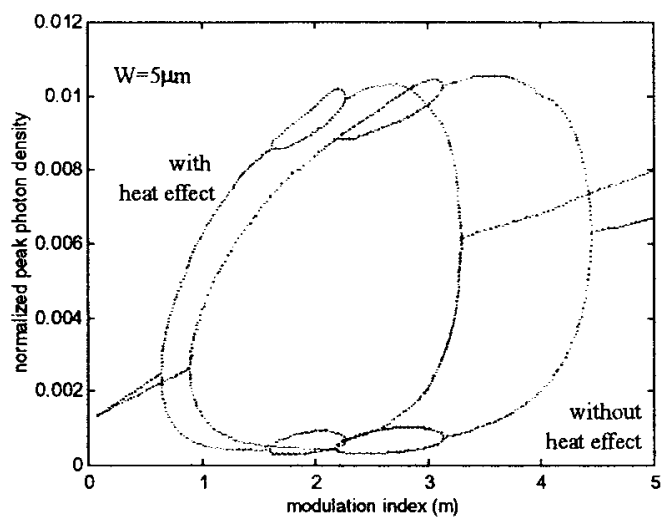

Fig. 8. Influence of the self-heating effect on the bifurcation diagram of VCSEL's with $W=5 \mu \mathrm{m}$ and $f_{m}=4 \mathrm{GHz}$.

of the dependence of the differential gain in $f_{r}$ on the selfheating temperature. Fig. 8 shows the bifurcation diagrams $P_{\text {npeak }}$ versus $m$ for devices with and without the self-heating effect taken into consideration. The self-heating effect can be ignored in the calculation by setting (3) to zero and the steadystate effective temperature to $300 \mathrm{~K}$ for the whole range of $m$. In the calculation, a device with $W=5 \mu \mathrm{m}$ is used and $P_{s}$ is set to $1.2 \times 10^{15} \mathrm{~cm}^{-3}$ (i.e., $0.32 \mathrm{~mW}$ ). It is observed that the entire bifurcation diagram shifts to the right-hand side if the self-heating effect is neglected in the calculation. This is because the differential gain as well as $f_{r}$ are overestimated in the calculation.

As we can see, although the influence of the self-heating effect is less important than the size-dependent parameters $P_{s}$ and $f_{r}$, the bifurcation diagram also changes significantly. In this case, period doubling may not be observed for $m \leq 1$ if the self-heating effect is neglected in the calculation.

\section{SimUlation ResUlts-MOdULATION AND OPTICAL FEEDBACK}

In this section, the modulation response of VCSEL's under large-signal modulation and external optical feedback is studied. The influence of $W$ and $R_{\text {ext }}$ on the chaotic behavior of VCSEL's is investigated. It is assumed that the steady-state output power and $f_{m}$ of lasers are set to $0.32 \mathrm{~mW}$ and $4 \mathrm{GHz}$, respectively. The Langevin noise sources are also considered in the calculation. It can be shown that devices with small $W$ suppress higher order bifurcations and chaos even under the influence of external optical feedback.

Fig. 9(a) shows the bifurcation diagram of $P_{\text {npeak }}$ versus $m$. The values of $W$ and $R_{\text {ext }}$ are set to $3.5 \mu \mathrm{m}$ and $10^{-3}$, respectively, in the calculation. The corresponding transient behavior of $P_{\text {norm }}$ at $m=4$ is shown in Fig. 9(b). It is observed that only a single period oscillation is excited for the whole range of $m$, but this bifurcation diagram is slightly thickened due to the noise and external optical feedback. Fig. 10 shows the bifurcation diagram of $P_{\text {npeak }}$ versus $m$ for a device with $W$ and $R_{\text {ext }}$ equal to $5 \mu \mathrm{m}$ and $10^{-3}$, respectively. It is observed that, as $m$ increases, the transient response of the laser evolves from a single period oscillation $(m<0.6)$ to period-doubling bifurcation $(0.6<m<3.2)$ and then to chaos $(m>3.2)$. The influence of $R_{\text {ext }}$ on the bifurcation 


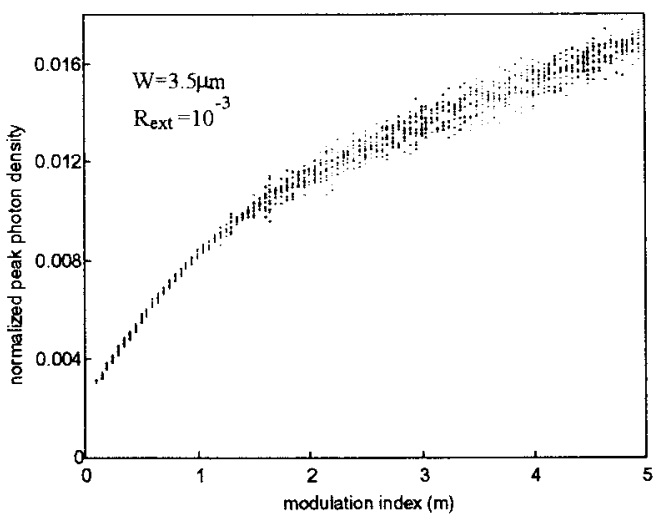

(a)

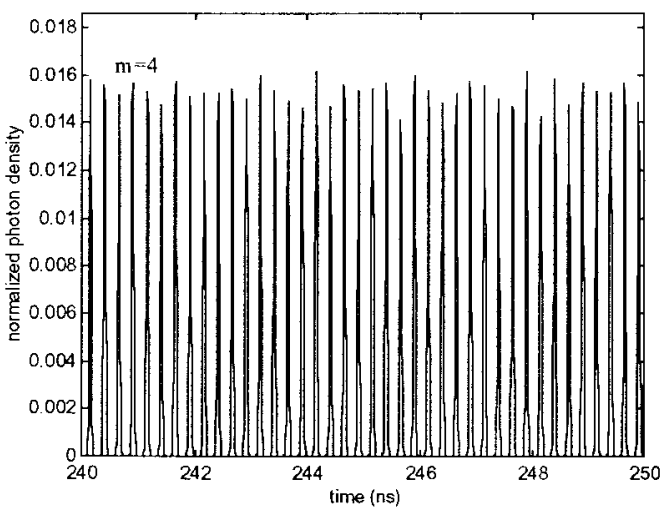

(b)

Fig. 9. Modulation response of VCSEL with $W=5 \mu \mathrm{m}, f_{m}=4 \mathrm{GHz}$ and $R_{\text {ext }}=10^{-3}$. (a) Bifurcation diagram of normalized peak photon density versus modulation index. (b) Transient response of normalized photon density at $m=4$.

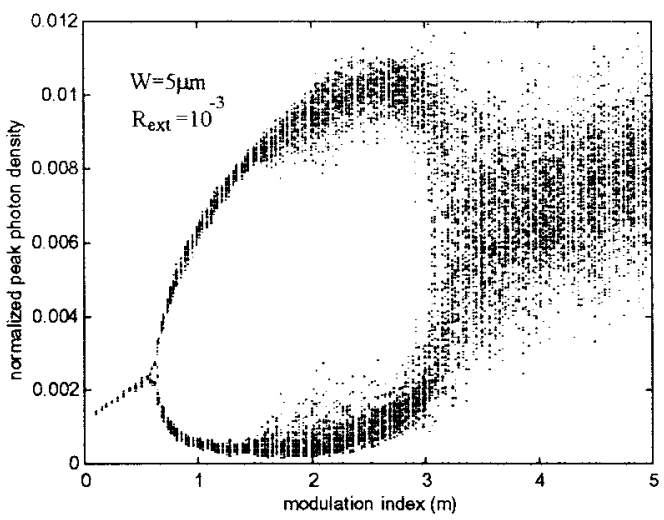

Fig. 10. Bifurcation diagram of normalized peak photon density versus modulation index for a VCSEL with $W=5 \mu \mathrm{m}, f_{m}=4 \mathrm{GHz}$, and $R_{\text {ext }}=10^{-3}$.

diagram of $P_{\text {npeak }}$ versus $m$ is also analyzed. Fig. 11 shows the bifurcation diagram $P_{\text {npeak }}$ versus $m$ for devices with $W=5 \mu \mathrm{m}$ and $R_{\text {ext }}=5 \times 10^{-2}$. It is found that the output power goes to chaos at a smaller value of $m(m \geq 2.8)$. In addition, the magnitude of $P_{\text {npeak }}$ is larger than that given in Fig. 10.

Furthermore, it is noted that the influence of size-dependent parameters such as $P_{s}, f_{r}$, and $\beta$ on the bifurcation diagrams with external optical feedback is similar to that given in Section III except that the introduction of $R_{\text {ext }}$ enhances

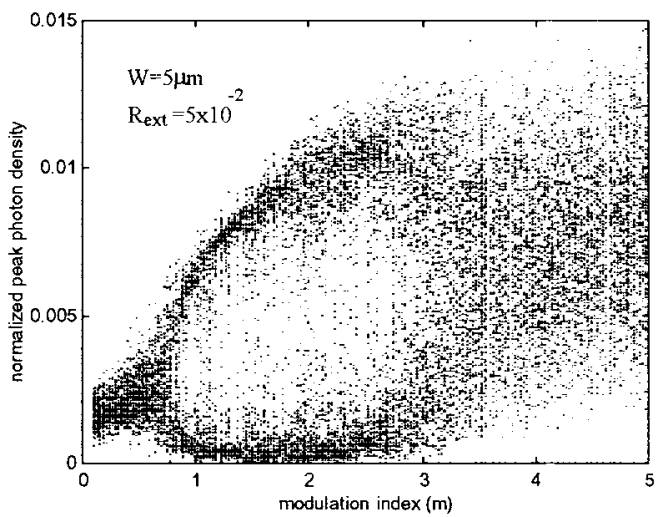

Fig. 11. Bifurcation diagram of normalized peak photon density versus modulation index for a VCSEL with $W=5 \mu \mathrm{m}, f_{m}=4 \mathrm{GHz}$, and $R_{\text {ext }}=5 \times 10^{-2}$.

chaotic characteristics. Therefore, the corresponding analysis is not repeated in this section. Hence, it is shown that devices with small $W$ suppress high order bifurcations and chaos even with external optical feedback.

\section{Simulation Results-Noise Characteristics}

In this section, the dependence of relative intensity noise (RIN) on $W$ of VCSEL's under external optical feedback $R_{\text {ext }}$ is studied. RIN is defined as

$$
\mathrm{RIN}=\left\langle\delta P^{2}\right\rangle /\langle P\rangle^{2}
$$

where $\langle P\rangle\left(=\overline{P_{0}(t)}\right)$ is the average photon density at steady state and $\delta P=P_{0}(t)-\langle P\rangle$. Langevin noise sources are also considered in the following calculation. It can be shown that devices with small $W$ suppress the onset of coherence collapse due to a high $P_{s}$.

Fig. 12 shows the RIN of VCSEL's versus $R_{\text {ext }}$ for devices with $W=3.5$ and $5 \mu \mathrm{m}$. The steady-state output power in both cases is set to $0.32 \mathrm{~mW}$. It is observed that, for a small value of $R_{\text {ext }}$ (i.e., $\leq 10^{-2}$ ), flat floors of RIN are observed for both devices. This is due to the domination of Langevin noise which is a nonchaotic behavior of lasers. On the other hand, a jump in RIN is observed at $R_{\text {ext }}=10^{-2}$ and $10^{-1}$ for devices with $W$ equal to 5 and $3.5 \mu \mathrm{m}$, respectively. Fig. 13(a) and (b) shows the bifurcation diagram of $P_{\text {npeak }}$ versus $R_{\text {ext }}$ and the transient response of $P_{\text {norm }}$ at $R_{\text {ext }}=10^{-1}$, respectively, for a device with $W=5 \mu \mathrm{m}$. It is observed that the sudden jump of RIN is due to the onset of coherence collapse by the external optical feedback. Furthermore, it can be shown that the transient response of devices with small $W$ (i.e., $3.5 \mu \mathrm{m}$ ) is similar to that given in Fig. 13 but the magnitude of $P_{\text {npeak }}$ is much smaller than that with large $W$ (i.e., $5 \mu \mathrm{m}$ ), especially inside the chaotic regime. Hence, a device with small $W$ has better immunity to the onset of coherence collapse.

In order to study the influence of $W$ on the onset of coherence collapse, the dependence of RIN on $P_{s}$ and $\beta$ is investigated. Fig. 14(a) shows the dependence of RIN on $P_{s}$ for a device with $W=5 \mu \mathrm{m}$. $P_{s}$ is allowed to vary from $8 \times 10^{14}$ to $1.2 \times 10^{15} \mathrm{~cm}^{-3}$. It is observed that the increase in $P_{s}$ increases the value of $R_{\text {ext }}$ for the onset of coherence collapse but reduces the value of RIN. Fig. 14(b) shows the dependence 


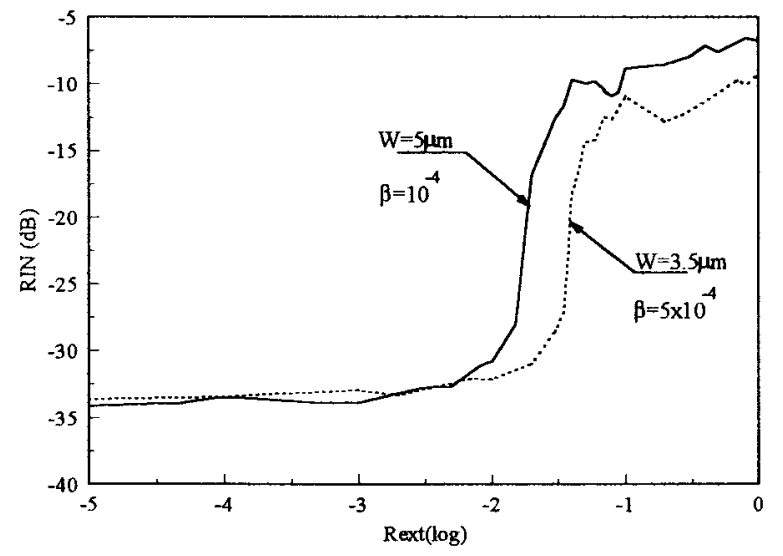

Fig. 12. RIN versus external reflectivity $R_{\text {ext }}$ for a VCSEL with $W$ equal to 3.5 and $5 \mu \mathrm{m}$.

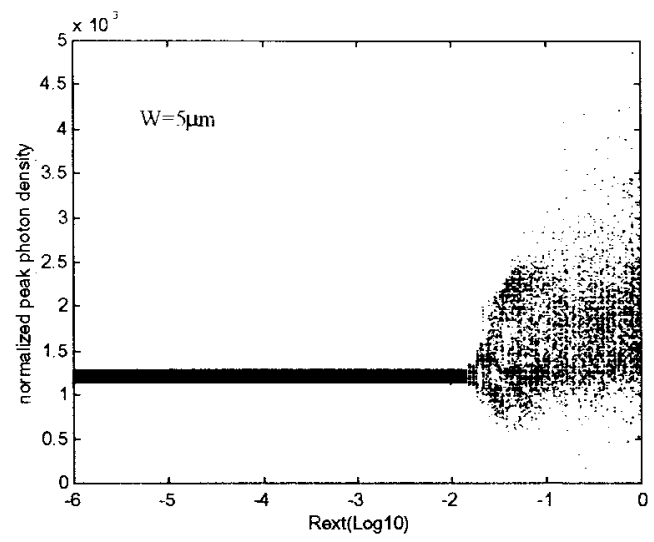

(a)

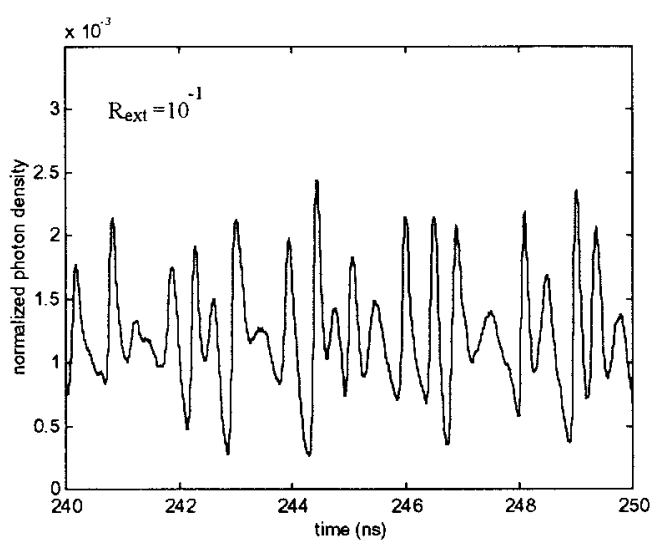

(b)

Fig. 13. The transient response of a VCSEL with $W=5 \mu \mathrm{m}$ under the influence of external optical reflection. (a) Bifurcation diagram of normalized peak photon density versus external reflectivity. (b) Transient response of normalized photon density at $R_{\text {ext }}=10^{-1}$.

of RIN versus $R_{\text {ext }}$ on the variation of $\beta$ (between $10^{-5}$ and $10^{-3}$ ) for a device with $W=5 \mu \mathrm{m}$. It is noted that the large value of $\beta$ enhances RIN but has a negligible influence on the onset of coherence collapse. Therefore, the onset of coherence collapse in VCSEL's is mainly due to $P_{s}$ (which is a function of $W$ ) but is independent of $\beta$. Therefore, we can conclude that VCSEL's with small $W$ will have better immunity to the onset of coherence collapse due to the high value of $P_{s}$.

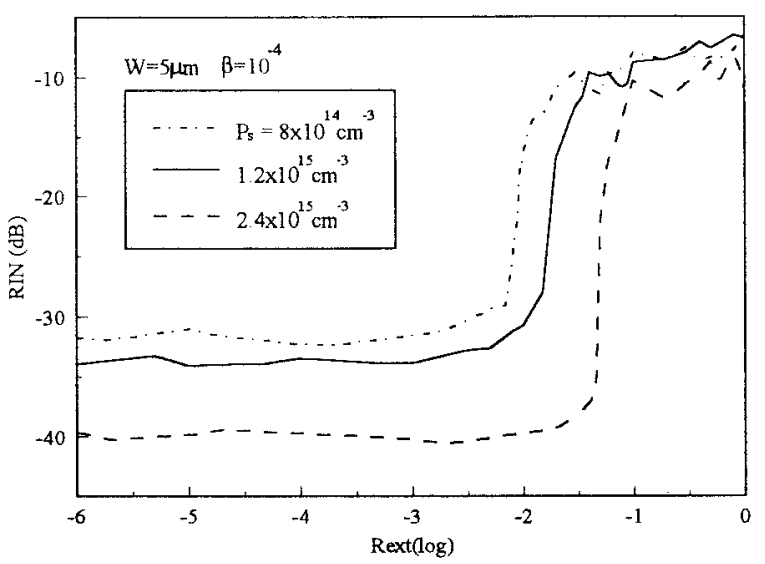

(a)

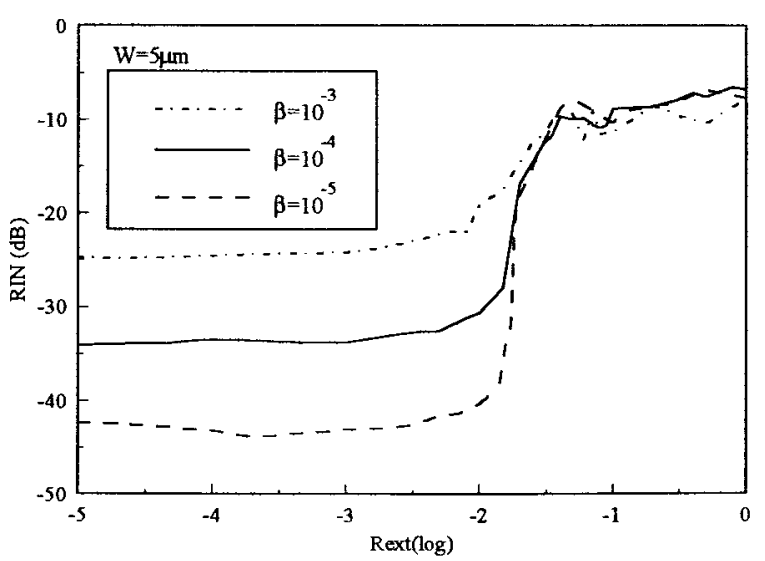

(b)

Fig. 14. RIN versus external reflectivity $R_{\text {ext }}$ for VCSEL with $W$ equal to $5 \mu \mathrm{m}$. (a) Photon density $P_{s}$ varies from $8 \times 10^{14}$ to $2.4 \times 10^{15} \mathrm{~cm}^{-3}$. (b) Spontaneous emission factor $\beta$ varies from $10^{-3}$ to $10^{-5}$.

\section{Simulation Results-ThiRd Harmonic Distortion}

The chaotic behavior of VCSEL's under large-signal modulation is studied extensively in the above sections. It is found that devices with small $W$ have better immunity to higher order bifurcations and chaos. On the other hand, it is noted that VCSEL's with small $W$ have better smallsignal characteristics such as wider AM bandwidth and lower second harmonic distortion than those with a large value of $W$ [3]. In addition, third-order harmonic distortion (THD) is an important factor in determining the performance of analog communication systems. In order to complete the picture on the small-signal modulation response of VCSEL's, the dependence of THD on $W$ is studied in this section.

The THD of VCSEL's is defined as

$$
\text { THD }=10 \cdot \log _{10}\left(P_{\text {third }} / P_{\text {first }}\right)
$$

where $P_{\text {first }}$ and $P_{\text {third }}$ are the first and third harmonic components of the output power signal. In the calculation, it is assumed that the steady-state power of lasers is maintained around $0.32 \mathrm{~mW}$ with $f_{m}$ varying between 2 and $5 \mathrm{GHz}$. $P_{\text {first }}$ and $P_{\text {third }}$ can be calculated by Fourier transform of the modulated output power signal. Fig. 15 shows the THD versus $m$ for devices with $W=3.5$ and $5 \mu \mathrm{m}$. It is observed that, for small-signal modulation (i.e., $m<0.05$ ), devices with small $W$ have a lower THD than those for large $W$ for 


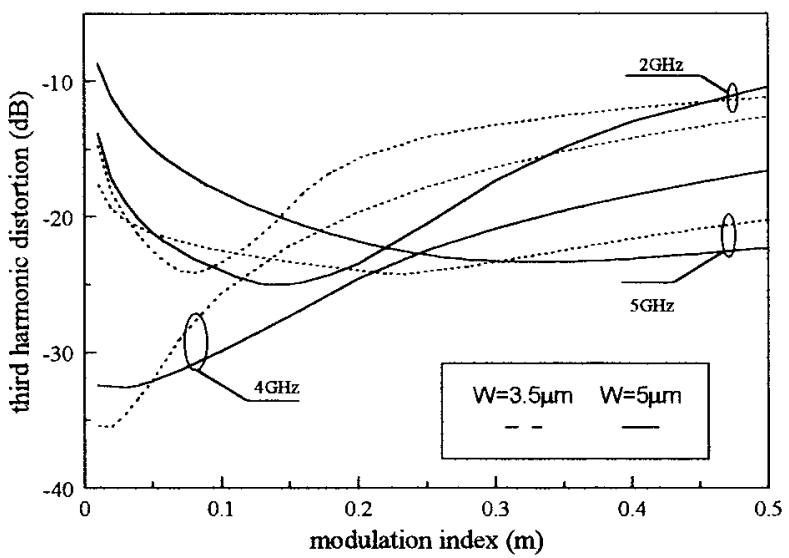

Fig. 15. THD versus modulation index $m$ for devices with $W$ equal to 3.5 and $5 \mu \mathrm{m}$ at different modulation frequencies.

all $f_{m}$. In fact, using small-signal analysis [3], one can show analytically that THD is inversely proportional to $f_{r}$ as well as $P_{s}$. Therefore, small devices have lower THD due to the high value of $f_{r}$ and $P_{s}$. However, for large $m$ (i.e., $>0.05$ ), the value of THD depends on $W$ as well as $m$.

\section{DISCUSSION AND CONCLUSIONS}

The modulation response of VCSEL's with $W=2.5 \mu \mathrm{m}$ is not shown in the above analysis. This is because $f_{r}$ is overestimated by the rate-equation model due to the existence of the gain saturation effect and the modulation response of VCSEL's may deviate from the real solution. If the gain saturation effect is omitted in the calculation, it can be shown that the device exhibits stable single-period oscillation even under large-signal modulation and external optical feedback. The influence of the gain saturation effect can be estimated approximately by increasing the magnitude of $\varepsilon$ to an unrealistic large value (e.g., $2 \times 10^{-16} \mathrm{~cm}^{3}$ ) such that the calculated value of $f_{r}$ can be closer to the experimental result. In this case, higher order bifurcations and chaos are excited under largesignal modulation. Nevertheless, the gain saturation effect can be neglected in the calculation for devices with large $W$ due to the relatively low value of $P_{s}$. Therefore, the above analysis is applicable to VCSEL's with $W \geq 3.5 \mu \mathrm{m}$.

In conclusion, the influence of $\bar{W}$ on the modulation response of VCSEL's is investigated using a single-mode rateequation model. The following results are found.

- VCSEL's with small $W$ (i.e., $3.5 \mu \mathrm{m}$ ) exhibit strong immunity to higher order bifurcations and chaos even under large-signal modulation and external optical feedback. This is because the nonlinear response is suppressed by high $f_{r}$ as well as $P_{s}$ in VCSEL's. In addition, a large value of $\beta$ acts as a damping factor in the rate equations to suppress any unstable performance of lasers [6]. Therefore, devices with small $W$ maintain a linear response under large-signal modulation and external optical feedback.

- The noise properties of VCSEL's subject to external optical feedback are studied via the calculation of RIN. It is found that the level of $R_{\text {ext }}$ for the onset of coherence collapse increases with the reduction of $W$ but $\beta$ has a negligible influence on the transition to chaos. This is because a high value of $P_{s}$ in small devices suppresses the onset of coherence collapse.

- The small-signal modulation response (i.e., $m<0.05$ ) of VCSEL's is also analyzed through the study of THD. It is shown that VCSEL's with a small $W$ have lower THD than devices with large $W$. This is because $f_{r}$ is inversely proportional to THD and devices with small $W$ have a higher $f_{r}$ than those with a large one.

Hence, VCSEL's with small $W$ have strong immunity to any instability which arises from small- or large-signal modulation as well as external optical feedback.

\section{REFERENCES}

[1] M. G. Peters, D. B. Young, F. H. Peters, J. W. Scott, B. J. Thibeault, and L. A. Coldren, "17.3\% peak wall plug efficiency vertical cavity surface emitting lasers using lower barrier mirrors," IEEE Photon. Technol. Lett., vol. 6, pp. 31-33, Jan. 1994.

[2] J. W. Scott, R. S. Geels, S. W. Corzine, and L. A. Coldren, "Modeling temperature effects and spatial hole burning to optimize vertical cavity surface emitting laser performance," IEEE J. Quantum Electron., vol. 29, pp. 1295-1308, 1993.

[3] S. F. Yu, W. N. Wong, P. Shum, and E. H. Li, "Theoretical analysis of modulation response and second harmonic distortion in vertical cavity surface emitting lasers," IEEE J. Quantum Electronics, vol. 32, pp. 2139-2147, Dec. 1996.

[4] C. H. Lee, T. H. Yoon, and S. Y. Shin, "Period doubling and chaos in a directly modulated laser diode," Appl. Phys. Lett., vol. 46, no. 1, pp. 95-97, 1985

[5] M. Tang and S. Wang, "Simulation studies of the dynamic behavior of semiconductor lasers with auger recombination," Appl. Phys. Lett., vol. 50, no. 26, pp. 1861-1863, 1987.

[6] Y. Hori, H. Serizawa, and H. Sato, "Chaos in a directly modulated semiconductor laser," J. Opt. Soc. Amer. B, vol. 5, no. 5, pp. 1128-1133, 1988.

[7] E. Hemery, L. Chusseau, and J. M. Lourtioz, "Dynamic behaviors of semiconductor lasers under strong sinusoidal current modulation: Modeling and experiments at $1.3 \mu \mathrm{m}$," IEEE J. Quantum Electron., vol. 26, pp. 632-641, Apr. 1990

[8] H. F. Liu and W. F. Ngai, "Nonlinear dynamics of a directly modulated $1.55 \mu \mathrm{m}$ InGaAsP distributed feedback semiconductor lasers," IEEE J. Quantum Electron., vol. 29, pp. 1668-1675, June 1993.

[9] G. Carpintero and H. Lamela, "Influence of noise in the route to chaos of directly modulated semiconductor lasers," J. Appl. Phys., vol. 82, no. 6, pp. 2766-2772, 1997.

[10] G. Shtengel, H. Temkin, T. Uchida, M. Kim, P. Brusenbach, and C. Parsons, "Spontaneous emission factor and its scaling in vertical cavity surface emitting lasers," Appl. Phys. Lett., vol. 64, no. 9, pp. 1062-1064, 1994.

[11] Y. G. Zhao, J. G. McInerney, and R. A. Morgan, "Measurement of spontaneous emission factor for vertical cavity surface emitting lasers," IEEE Photon. Technol. Lett., vol. 7, pp. 1231-1233, Nov. 1995.

[12] J. H. Shin, Y. G. Ju, H. E. Shin, and Y. H. Lee, "Spontaneous emission factor of oxidized vertical cavity surface emitting lasers for the measured below threshold cavity loss," Appl. Phys. Lett., vol. 70, no. 18, pp. 2344-2346, 1997.

[13] H. M. Chen, K. Tai, K. F. Huang, Y. H. Kao, and J. D. Wynn, "Instability in surface emitting lasers due to external optical feedback," J. Appl. Phys., vol. 73, no. 1, pp. 16-20, 1993.

[14] S. Jiang, M. Dagenais, and R. A. Morgan, "Spectral characteristics of vertical cavity surface emitting lasers with strong external optical feedback," IEEE Photon. Technol. Lett., vol. 7, pp. 739-741, July 1995.

[15] U. Fiedler and K. J. Ebeling, "Design of VCSEL's for feedback insensitive data transmission and external cavity active mode locking," IEEE J. Select. Topics Quantum Electron., vol. 1, pp. 442-450, March 1995.

[16] L. N. Langley and K. A. Shore, "Effect of optical feedback on the noise properties of vertical cavity surface emitting lasers," Proc. Inst. Elect. Eng., pt. J, vol. 144, no. 1, pp. 34-38, 1997.

[17] J. Y. Law and G. P. Agrawal, "Effects of optical feedback on static and dynamic characteristics of vertical cavity surface emitting lasers," IEEE J. Select. Topics Quantum Electron., vol. 3, pp. 353-358, March 1997.

[18] P. S. Spencer, C. R. Mirasso, and K. A. Shore, "Effect of strong optical feedback on vertical cavity surface emitting lasers," IEEE Photon. Technol. Lett., vol. 10, pp. 191-193, Feb. 1998. 


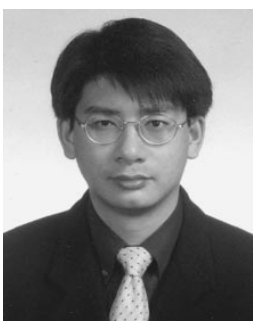

S. F. Yu received the B.Eng. degree (first class Honors) in electronic engineering from London University, University College, U.K., in 1990 and the $\mathrm{Ph} . \mathrm{D}$. degree in optoelectronic engineering from Cambridge University, Robinson College, U.K., in 1993.

He joined the Department of Electronic Engineering, Sha Tin Technical Institute, Hong Kong, as a part-time Lecturer in 1993. In 1994, he joined the Department of Electrical and Electronic Engineering, the University of Hong Kong, where he was a Lecturer. Since 1996, he has been an Assistant Professor in the same department of the University of Hong Kong. His main research interest includes the fundamental study, optimization, and practical applications of semiconductor lasers. He currently conducts the development of highperformance semiconductor lasers using diffused quantum- well materials for application in long-haul optical fiber communication systems. He is also involved in the development of novel mid-infrared vertical- cavity lasers using quantum cascade architecture and their applications in the measurement of gas and liquid concentrations. He has published over 60 international technical papers including invited conference and journal papers as well as two book chapters. He is also a member of the executive committee and is the Seminar Program Chair of the seminar and meeting committee of the SPIE Hong Kong Chapter. His biography has published in the 4th edition of Who's Who in Science and Engineering (Marquis, USA), the 25th edition of the Dictionary of International Biography, and the 17th edition of the International Directory of Distinguished Leadership.

Dr. Yu was awarded the traditional Departmental Prize by the Department of Electronics and Electrical Engineering, University College London, in the final year degree examination. He was a Fellow and Honorary Scholar of Cambridge Commonwealth Trust Society. He also held a Croucher Foundation scholarship and an overseas research student award while studying for the doctoral program. He is a member of SPIE. 